\title{
Research on Method for Monitoring Service Life of Hot-End Components in Gas Turbine based on Condition Analysis
}

\author{
Yong-chun LIANG ${ }^{1}$, Zhang-jun HUANG ${ }^{2}$, a ${ }^{*}$, Lu-ping $\mathrm{LI}^{2}$, Xue-zhi Tang ${ }^{2}$ and \\ Yi-qian $\mathrm{XIAO}^{2}$ \\ ${ }^{1}$ Electric Power Research Institute of Guangdong Power Grid Company, Guangzhou 510080, \\ China \\ ${ }^{2}$ School of Energy and Power Engineering, Changsha University of Science and Technology, \\ Changsha 410014, China \\ ahzhangjun1103@163.com
}

Keywords: gas turbine; hot-end components; service life loss; monitoring; power load.

Abstract. The service life of hot-end components in gas turbine for gas-fired power plant is hard to detect and evaluate. The relationship between temperature characteristics of hot-end components and the power load is established using the basic theory of gas turbine power cycle. The operating mode of gas turbine unit is distinguished and determined through the use of electricity load and the rotor rotational speed. The equivalent operation hours and service life loss of the gas turbine and its hot-end components under various conditions are obtained by calculation. Research results show that the method for monitoring service life of hot-end components in gas turbine based on power load analysis can realize the real-time monitoring and assessment of the service life loss and residual life for hot-end components through the measuring parameters from the unit control system, which is advantageous for timely and accurately assessment of the reliability and cumulative life loss of hot-end components, and so to provide the reliable operation of the gas turbine hot-end components.

\section{Introduction}

With the continuous development and the widespread application of modern gas turbine technology, research on key technology and whole design of gas turbine with completely independent intellectual property rights has attached more and more importance $[1,2]$. To sustain high levels of performance and reliability of this equipment, diagnostics and maintenance planning have also become increasingly important. In order to assess the "health" of engine components, condition monitoring techniques have been adopted recently [3].

There were two standard methods of turbine engine condition monitoring. One is to compare recorded engine parameters with operational limits from engine manufacturers, and the other is to monitor the trends in engine data so to detect sudden shifts in operational behavior. The two standard methods use directly engine data for condition monitoring, however these methods can be sensitive to the quality and quantity of example data. Some advanced methods for turbine engine condition monitoring were brought out, such as CASE-based reasoning, the expert systems with fuzzy logic rules and neural network methods [4-7]. However, the CASE-based reasoning approach was limited by the variation in operational behavior between classes of engine, and the expert systems were attempted to improve the generality by the combination of databases from multiple engines. Furthermore, frequency-domain technique has been used for the faults diagnosis of turbine engine and the estimation of parametric and nonparametric models of the fuel feed to shaft speed dynamics of a twin-shaft gas turbine engine [8], and the acoustic emission (AE) technique was also used for in-service condition monitoring of a gas turbine under various conditions [9].

Hot-end components in a gas turbine are the components of channel through which the high temperature gas flows during the gas turbine running. Huge challenges are put forward for the maintenance, performance and service life analysis of the hot-end components due to the harsh environment and conditions in gas turbine. It is of great importance to predict the life exhaustion and remaining life of hot-end components subjected to complex loading spectra via effective condition 
monitoring and life assessment method. From the point of view of monitoring parameters, established condition monitoring techniques for turbines generally involve vibration, oil-debris, temperature and pressure monitoring, however they are indirect measurement methods which often require significant fault progression before detection is possible. To date, there is few published work related to the load-monitoring of large gas turbine engines. Due to the convenience and practicability of load-monitoring method, the life exhaustion and remaining life of hot-end components in gas turbines are analyzed and assessed by use of the load-monitoring and condition analysis.

\section{Calculation model of service life under stable load}

A gas turbine engine typically consists of a compressor section, a combustion chamber and a rear-mounted turbine. The compressor draws in and pressurizes air, and then delivers it to the combustion chamber, where fuel and compressed air are mixed and ignited. The hot gas expands rapidly and provides motive force in the turbine. The compressor section is driven by the turbine via a connected shaft between them.

The power from expansion of flue gas in turbine can be calculated as

$$
P_{t}=Q\left(h_{3}-h_{4}\right)=Q c_{p}\left(T_{3}-T_{4}\right)
$$

Where $P_{t}$ is the ideal output power of gas turbine, $Q$ is the gas flow rate, $h_{3}$ is the enthalpy of flue gas at the entry of gas turbine, $h_{4}$ is the enthalpy of exhaust gas, $c_{p}$ is the specific heat at constant pressure of the flue gas, $T_{3}$ and $T_{4}$ are thermodynamic temperature of the flue gas at inlet and outlet of the turbine respectively.

The power consumption of the compressor can be calculated as

$$
P_{c}=Q\left(h_{2}-h_{1}\right)=Q c_{p}\left(T_{2}-T_{1}\right)
$$

Where $P_{c}$ is the ideal power consumption of the compressor, $h_{1}$ and $h_{2}$ are the enthalpy of air at inlet and outlet of the compressor respectively, $T_{1}$ and $T_{2}$ are thermodynamic temperature of air at inlet and outlet of the compressor respectively.

The net output power of the gas turbine can be calculated as

$$
\begin{aligned}
P_{n e t} & =Q\left[\left(h_{3}-h_{4}\right)-\left(h_{2}-h_{1}\right)\right] \\
& =Q c_{p} T_{3}\left(1-\frac{T_{4}}{T_{3}}-\frac{T_{2}}{T_{3}}+\frac{T_{1}}{T_{3}}\right) \\
& =Q c_{p} T_{3}\left(1-\pi^{\frac{1-k}{k}}-\frac{\pi^{\frac{k-1}{k}}}{\tau}+\frac{1}{\tau}\right)
\end{aligned}
$$

Where $P_{n e t}$ is the net output power of the gas turbine, $\tau$ is the temperature rise ratio of the gas turbine, $\pi$ is the pressure rise ratio of the compressor, $k$ is the adiabatic exponent of the working gas in the gas turbine.

The output power of the electrical generator driven by the gas turbine can be calculated as

$$
P_{e l}=\eta_{g} Q c_{p} T_{3}\left(1-\pi^{\frac{1-k}{k}}-\frac{\pi^{\frac{k-1}{k}}}{\tau}+\frac{1}{\tau}\right)
$$

Where $P_{e l}$ is the output power of the electrical generator, and $\eta_{g}$ is the efficiency of the electrical generator driven by the gas turbine.

The thermodynamic temperature $T_{3}$ in Eq. 4 reflects the temperature of hot-end components, and $P_{e l}$ in Eq. 4 is the output power of the electrical generator, so the internal relation between temperature level of hot-end components and the output power of the electrical generator is set up through Eq. 4, 
which provides a theoretical basis for the monitoring service life of hot-end components based on the condition and load analysis.

The service life of hot-end components can be quantified with equivalent operation hours (EOH). According to the average working temperature, the hot-end components are divided into two categories, i.e. class I with higher average working temperature and class II with lower average working temperature. The class I hot-end components include the flame tube, the transition section, the interconnector, the first stage of static blades, the first stage of moving blades, etc. The class I hot-end components include the moving blade, static blades and complex ring at from the second stage to the fourth stage, etc.

The electricity load factor of gas turbine generator unit needs to be determined according to the real output power and the rated output power of the electrical generator. The $\mathrm{EOH}$ of hot-end components under stable load can be calculated as

$$
T_{P}^{i}=\alpha_{p} \beta_{P} T_{P}
$$

Where $P_{p}^{i}$ is the equivalent operation hours of hot-end components under stable load, $\alpha_{p}$ is the service-life-difference coefficient of the of hot-end components in the gas turbine, $\beta_{p}$ is the electric load factor, and $T_{p}$ is the actual operation hours of the gas turbine. For class I and class II hot-end components, can $\alpha_{p}$ be set to $1.0 ; \beta_{p}$ can be set to 1.1 when $P_{e l}$ is larger than 0.8 times of $P_{e l, 0}$ that denotes the rated output power of the electrical generator, otherwise $\beta_{p}$ can be set to 1.0 .

\section{Calculation model of service life under unstable load}

In the process of practical operation of gas turbine, the service life and reliability of hot-end components are closely related to the actual operation conditions of gas turbine. The analysis and recognition of operation conditions are shown in Table 1, where $n$ is the engine revolutions per minute $(\mathrm{rpm}), n_{0}$ is the engine rated $\mathrm{rpm}, P_{e l}$ is the output power of the electrical generator, $P_{e l, 0}$ is the rated output power of the electrical generator, $t$ is the elapsed time.

Table 1 Analysis of operation conditions using process parameters

\begin{tabular}{|c|c|c|}
\hline Item No. & Process parameters & Operation conditions \\
\hline 1 & $\left(n / n_{0}\right)<0.95,\left|\mathrm{~d} P_{e l} / \mathrm{d} t\right| \in[0.005,0.08)\left(P_{e l, 0} / \mathrm{min}\right)$ & On-off operation \\
\hline 2 & $\left(n / n_{0}\right) \in[0.95,1.05),\left|\mathrm{d} P_{e l} / \mathrm{d} t\right| \in[0.05,0.08)\left(P_{e l, 0} / \mathrm{min}\right)$ & Fast-change load \\
\hline 3 & $\left(n / n_{0}\right) \in[1.05,1.1), \mathrm{d} P_{e l} / \mathrm{d} t<-0.08\left(P_{e l, 0} / \mathrm{min}\right)$ & Load shedding \\
\hline 4 & $\left(n / n_{0}\right) \geq 1.1, \mathrm{~d} P_{e l} / \mathrm{d} t<-0.08\left(P_{e l, 0} / \mathrm{min}\right)$ & Emergency stop \\
\hline
\end{tabular}

The EOH of hot-end components under unstable load can be calculated according the operation conditions. The EOH of hot-end components at on-off operation condition can be calculated as

$$
T_{c}^{i}=\alpha \beta_{c} N_{c}
$$

Where $T_{c}^{i}$ is the EOH of hot-end components at on-off operation condition, $\alpha$ is the service-life-difference coefficient of hot-end components in the gas turbine, $\beta_{c}$ is the conversion factor of EOH for on-off operation, and $N_{c}$ is the number of on-off operation.

The EOH of hot-end components at fast-change load condition can be calculated as

$$
T_{k}^{i}=\alpha \beta_{k} N_{k}
$$

Where $T_{k}{ }_{k}$ is the EOH of hot-end components at fast-change load condition, $\alpha$ is the service-life-difference coefficient of hot-end components, $\beta_{k}$ is the conversion factor of $\mathrm{EOH}$ for fast-change load condition, and $N_{k}$ is the number of fast-change load condition.

The EOH of hot-end components at load shedding condition can be calculated as

$$
T_{r}^{i}=\alpha \beta_{r} N_{r}
$$


Where $T_{r}^{i}$ is the EOH of hot-end components at load shedding condition, $\alpha$ is the service-life-difference coefficient of hot-end components, $\beta_{r}$ is the conversion factor of $\mathrm{EOH}$ for load shedding condition, and $N_{r}$ is the number of load shedding condition.

The EOH of hot-end components at emergency stop condition can be calculated as

$$
T_{j}^{i}=\alpha \beta_{j} N_{j}
$$

Where $T_{j}$ is the $\mathrm{EOH}$ of hot-end components at emergency stop condition, $\alpha$ is the service-life-difference coefficient of hot-end components, $\beta_{j}$ is the conversion factor of $\mathrm{EOH}$ for emergency stop condition, and $N_{j}$ is the number of emergency stop condition.

The total EOH of hot-end components are the sum of EOH under various operation conditions, i.e.

$$
T_{E}=\sum T_{P}^{i}+\sum T_{c}^{i}+\sum T_{r}^{i}+\sum T_{j}^{i}+\sum T_{k}^{i}
$$

Where $T_{E}$ denotes the total EOH of hot-end components.

\section{Engineering application}

A class $\mathrm{F}$ gas turbine in a domestic LNG power plant is chosen as the measured object for our engineering application, and the service-life monitoring system for the corresponding parameter and hot-end components is established. The overall structure of the service-life monitoring system is shown in Fig. 1.

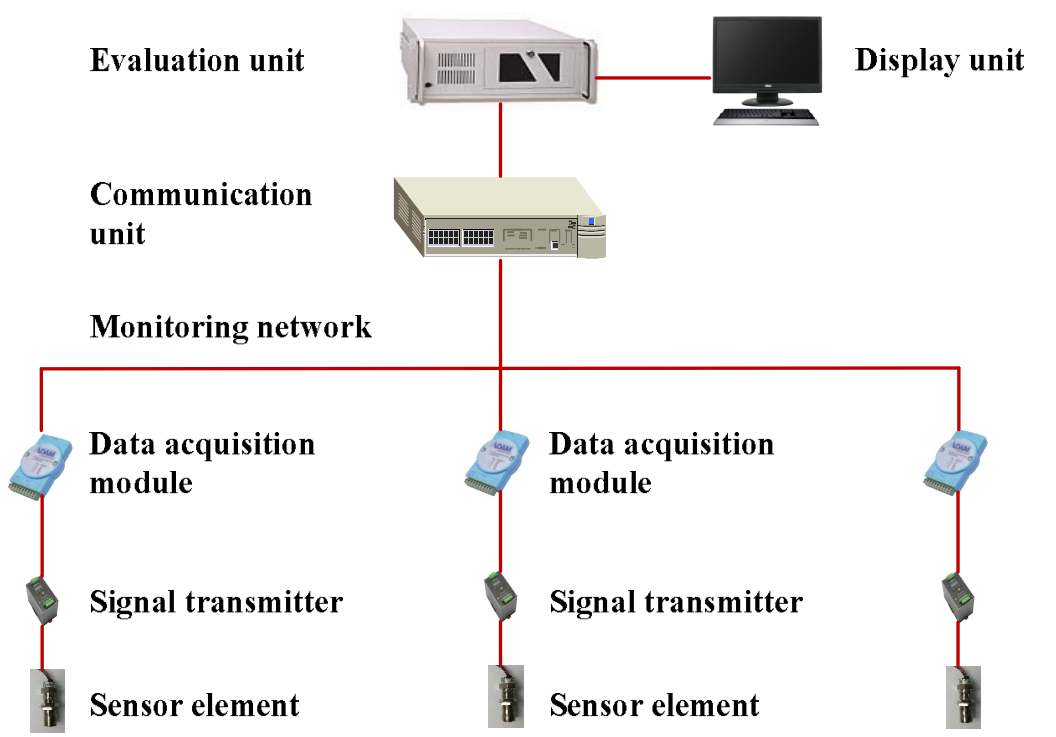

Figure 1. The overall structure of the service-life monitoring system

The service-life monitoring system consists of sensor elements, signal transmitters, data acquisition modules, the monitoring network, units for communication, evaluation and display. Sensor elements get the operation parameters and sent them to the evaluation unit via signal transmitters, data acquisition modules, the monitoring network and communication unit. The evaluation unit performs the parameter analysis and calculation using the proposed method above for the service-life assessment of hot-end components in the gas turbine.

Using the operation parameters and the measured data from the service-life monitoring system, the service-life of first-stage-static blades is analyzed and calculated in Table 2.

Table 2. The service-life calculation of first-stage-static blades

\begin{tabular}{|c|c|c|c|}
\hline Operation parameters & Measured number & Calculation factors & EOH $[\mathrm{h}]$ \\
\hline Stable load & $2500[\mathrm{~h}]$ & $\alpha_{p}=1.0, \beta_{p}=1.0$ & 2500 \\
\hline On-off operation & 120 & $\alpha=20, \beta_{c}=0.1$ & 240 \\
\hline Fast-change load & 82 & $\alpha=20, \beta_{k}=0.2$ & 328 \\
\hline Load shedding & 13 & $\alpha=20, \beta_{r}=3.0$ & 780 \\
\hline Emergency stop & 4 & $\alpha=20, \beta_{j}=3.0$ & 240 \\
\hline
\end{tabular}


The total EOH of the first-stage-static blades are 4088 hours according to results in Table 1 and Eq. 10. The relative error is less than $5 \%$ by comparison the calculated results with the data 4290 hours from the manufacturer, which indicates the high reliability and accuracy of the service-life monitoring method and system for hot-end components in the gas turbine.

\section{Conclusions}

A service life monitoring method for hot-end components in the gas turbine is put forward through the analysis of gas turbine power cycle and the subsequent establishment of the relationship between temperature characteristics of hot-end components and the power load. The electricity load and the rotor rotational speed are used for the analysis and recognition of operation conditions, which is utilized to calculate the equivalent operation hours and service-life of hot-end components under various operation conditions. Results of the engineering application show that the service-life monitoring method for hot-end components in the gas turbine is reliable and accurate, which can provide some necessary reference and technical supports to master and assess the service-life accumulation and the reliability of hot-end components in gas turbines.

\section{Acknowledgments}

The research is supported by Science and Technology Project of Guangdong Power Grid Corporation (No. K-GD2014-0583002-002), Science and Technology Project of Hunan Province (No. 2014GK3118).

\section{References}

[1] H. Jiang, J. Ren, X. Li, Q. Tan, Status and development trend of the heavy duty gas turbine, Proceedings of the CSEE, 29 (2014) 5096-5102. (In Chinese)

[2] N. Cai, R. Cui, K. Chen, H. Yang, H. Liu, Independent research and development for r0110 heavy duty gas turbine, Gas Turbine Technology, 3 (2014) 1-7. (In Chinese)

[3] V.P. Surender, R. Ganguli, Adaptive myriad filter for improved gas turbine condition monitoring using transient data, Journal of Engineering for Gas Turbines \& Power, 127 (2004) 479-489.

[4] A. Yousuf, W. Cheetham, Case-based reasoning for turbine trip diagnostics, Case-Based Reasoning Research and Development, Springer Berlin Heidelberg, 2012, pp. 458-468.

[5] M. Xiao, Y. Chen, S. Yao, L. Jiang, Research on expert system of gas turbine fault diagnosis based on fuzzy neural network, Journal of Jiangsu University of Science \& Technology, 4 (2009) 331-334. (In Chinese)

[6] A. Hamid, X. Chen, M.B. Menhaj, S. Raazesh, Artificial neural network-based system identification for a single-shaft gas turbine, Journal of Engineering for Gas Turbines \& Power, 9 (2013) 942-955.

[7] I. Loboda, Y. Feldshteyn, Polynomials and neural networks for gas turbine monitoring: a comparative study, International Journal of Turbo and Jet Engines, 28 (2010) 227-236.

[8] C. Evans, D. Rees, A. Borrell, Identification of aircraft gas turbine dynamics using frequency-domain techniques, Control Engineering Practice, 8 (2000) 1372-1378.

[9] R. M. Douglas, M.D. Jenkins, A.K. Frances, J.A. Steel, R.L. Reuben, P.A. Kew, Monitoring of gas turbine operating parameters using acoustic emission, Proceedings of 2004 EWGAE, 1 (2004) 389-396. 